\title{
ORIGINAL ARTICLE \\ The practice of spinal cord injury core data collection among Chinese physicians: a survey-based study
}

\author{
N Liu ${ }^{1}, \mathrm{Z}-\mathrm{W} \mathrm{Hu}{ }^{1}, \mathrm{M}-\mathrm{W}$ Zhou $^{1}$ and F Biering-Sørensen ${ }^{2}$
}

\begin{abstract}
Study design: This is a survey-based study.
Objective: To investigate the practice of spinal cord injury ( $\mathrm{SCl}$ ) core data collection by Chinese physicians to measure the extent and accuracy of routine collection of elements contained in the International Spinal Cord Injury Core Data Set (ISCICDS).

Setting: This study was conducted in a workshop in Peking University, China.

Methods: During an SCl workshop, a survey questionnaire was administered to 48 physicians from 20 provinces of China. The questions were developed on the basis of the data elements within the ISCICDS including the following issues: date of birth, injury, acute admission and inpatient discharge, total hospitalized days, gender, injury etiology, vertebral injury, associated injury, spinal surgery, ventilatory assistance and place of discharge. In addition, data collection practice on neurologic examinations including date, neurological level, injury severity and frequency of examination were involved.
\end{abstract}

Results: The self-reported practice of data collection regarding date of birth, acute admission and inpatient discharge, gender, vertebral injury, associated injury, spinal surgery and frequency of neurological examination are consistent with the information in the ISCICDS among the majority ( $\geqslant 76 \%)$ of physicians. However, only gender, vertebral injury, associated injury and spinal surgery are completely consistent. The consistency percentages of other data elements ranged from 39.5 to $66.8 \%$.

Conclusion: Apart from four data elements, which were collected consistently with the intention in the ISCICDS, the collection of other core data elements need to be documented according to the guidelines included in the ISCICDS to ensure consistency of practice among Chinese physicians and to support worldwide comparison of SCl data.

Suggestion: Only four data elements are collected in complete accordance with the ISCICDS by Chinese physicians. ISCICDS guidelines for the remaining elements need to be more rigorously adhered to in order to promote consistency and comparability of data. Spinal Cord (2015) 53, 658-662; doi:10.1038/sc.2014.217; published online 9 December 2014

\section{INTRODUCTION}

The initiative of International Spinal Cord Injury Data Sets was proposed by the International Spinal Cord Society (ISCoS) in collaboration with the American Spinal Injury Association (ASIA). ${ }^{1}$ Numerous data sets have been developed by specialized international working groups, and they have been released on the website of ISCoS (http://www.iscos.org.uk/international-sci-data-sets). The aim of developing International Spinal Cord Injury Data Sets is to gather consistent data elements to enable worldwide SCI data and outcomes to be assessed and compared. ${ }^{1}$

Among the available data sets, the International Spinal Cord Injury Core Data Set (ISCICDS) ${ }^{2}$ is central because it is essential for documenting basic information during the initial inpatient rehabilitation period in individuals with spinal cord injury (SCI). The ISCICDS, as well as training cases, is presented on the website of ISCoS (http://www.iscos.org.uk/resources/sitefiles/PageFile_20_coredataset.pdf). After receiving consent from the Executive Committee for the International SCI Standards and Data Sets, the official Chinese translation of ISCICDS following the translation guideline ${ }^{3}$ was performed and is now available on the website (http://www. iscos.org.uk/sitefiles/Chinese $\% 20$ translation\%20International\%20SCI
\%20Core\%20Data\%20Set $\% 20 \% 20$ Version $\% 201.0 \% 20 \% 20-\% 20 \%$ 20incl.\%20TRAINING\%20Cases.pdf).

By using the data collection form, the data within the ISCICDS can be collected in clinical settings. However, some data may already be documented in clinical practice, whereas others may not. If the method of data collection used in clinical practice is in accordance with the information in the ISCICDS, then such data may be retrieved retrospectively from the charts, whereas other data will have to be collected using the guidelines contained in the ISCICDS to ensure consistency across centers.

In this study, the practice of collecting information described in the ISCICDS in individuals with SCI performed by physicians in China was investigated. A questionnaire adapted from the data elements within the ISCICDS was used to allow comparison between current practice and that described in the ISCICDS. The results of this study will provide a preliminary overview of SCI core data collection among Chinese SCI physicians. On the basis of these results, recommendations regarding data collection across Chinese SCI centers will be elaborated to ensure that uniform data, comparable to other SCI centers worldwide, will be gathered.

${ }^{1}$ Department of Rehabilitation Medicine, Peking University Third Hospital, Beijing, China and ${ }^{2}$ Department for Spinal Cord Injuries, Glostrup University Hospital and Faculty of Health Sciences, University of Copenhagen, Copenhagen, Denmark

Correspondence: Professor M-W Zhou, Department of Rehabilitation Medicine, Peking University Third Hospital, No. 49 North Garden Road, Beijing 100191, China. E-mail: zhoumouwang@163.com

Received 9 June 2014; revised 20 October 2014; accepted 21 October 2014; published online 9 December 2014 


\section{MATERIALS AND METHODS}

In September 2013, 48 physicians from 20 of 31 Chinese provinces attended an SCI workshop in Peking University. All attendees were from the major hospitals with inpatient SCI rehabilitation in China, and represented the more populated provinces. The study was conducted during the workshop using a survey questionnaire about the data collection in clinical practice.

\section{Spinal Cord Injury Core Data Documentation Questionnaire}

The questions within the questionnaire (Appendix) were developed on the basis of the 20 data elements in the ISCICDS. Of these, four neurological data elements are to be collected at acute admission and at final inpatient discharge, respectively: that is, date of neurological examination, sensory level (SL), motor level (ML) and ASIA Impairment Scale (AIS). Other data elements include date of birth, injury, acute admission and inpatient discharge, total days hospitalized, gender, injury etiology, vertebral injury, associated injury, spinal surgery, ventilatory assistance and place of discharge.

Within the Spinal Cord Injury Core Data Documentation Questionnaire, the numbers of the first 12 questions correspond to the first 12 data elements within the ISCICDS. The remaining four questions are related to neurological data. To facilitate answering the questionnaire, the SL and the ML were combined into one question. In addition, a question regarding the frequency of neurological examinations was added to be able to report the number of, and the interval between, examinations from acute admission to final inpatient discharge. Thus, the 13th question is about the dates of the neurological examinations. The 14th and 15th questions are related to the level and severity of injury, respectively. The last question is added to indicate the frequency of the neurological examination.

The options within each question are developed to resemble the data documentation in clinical practice. In each question, one option corresponds to the information in the ISCICDS. Furthermore, some options may be briefer than in the ISCICDS, whereas other options may be more detailed. For example, in Q1 'In the chart, does it contain birth date information of individuals with SCI?', both option A and option B are briefer than the ISCICDS option C. In contrast, in Q10 'In the chart, how do you document spinal surgery of individuals with SCI?', both option B and option C are more detailed than the ISCICDS option A. As the more detailed option already includes the information required by ISCICDS, both the standard and the more detailed options were considered as being answered consistently with the ISCICDS.

If an option reflects all requirements of a data element in the ISCICDS, then this data element was asked in a single question. For example, when asking

Table 1 Percentages of the data collection practice among Chinese physicians consistent with the information in the ISCICDS

\begin{tabular}{lc}
\hline Data element & Percentage \% $(\mathrm{N}=48)$ \\
\hline Birth date & 66.7 \\
Injury date & 89.6 \\
Acute admission date & 79.2 \\
Final inpatient discharge date & 87.5 \\
Total days hospitalized & 39.5 \\
Gender & 100 \\
Injury etiology & 62.5 \\
Vertebral injury & 100 \\
Associated injury & 100 \\
Spinal surgery & 100 \\
Ventilatory assistance & 45.8 \\
Place of discharge & 54.2 \\
Date of neurological examination & 47.9 \\
Sensory/motor level & 68.8 \\
ASIA Impairment Scale (AIS) & 68.8 \\
Frequency of neurological examination & 83.3
\end{tabular}

Abbreviations: ASIA, American Spinal Injury Association; ISCICDS, International Spinal Cord Injury Core Data Set. about 'date of birth' in Q1, an option was developed to match the data element in ISCICDS, 'Yes, document as YYYYMMDD'. Otherwise, more subquestions are developed to ensure the completeness of the requirement of the data element. For example, when asking about 'place of discharge' in Q12, subquestions were customized. The initial question 'In the chart, do you document place of discharge of individuals with SCI?' checked whether the place of discharge is documented. Second, the question 'In the chart, will the following items be considered as place of discharge and documented for individuals with SCI?' was accompanied by a list of all potential places given in the ISCICDS. Finally, 'In the chart, do you document any other place of discharge different from those in 12-2 above in individuals with SCI?' was added to represent 'Others' in the ISCICDS. Each question and related subquestions corresponded to a single data element.

After the survey, the number of answers corresponding to the information in the ISCICDS to each question was counted (Table 1). For the data elements with a single question, the response could be analyzed directly—-that is, did the answer match the information in the ISCICDS. For the data elements with subquestions, only the responses from each subquestion were matched with the information in the ISCICDS for consistency.

\section{RESULTS}

The percentages of data elements collected consistently with the information in the ISCICDS are shown in Table 1. According to the result of this survey, four items were reported to be collected completely consistently with the information in the ISCICDS; these are gender, vertebral injury, associated injury and spinal surgery. The following four data elements exhibit above $75 \%$ consistency: injury date, acute admission date, final inpatient discharge date and frequency of neurological examination. Five data elements were reported to be lower than $75 \%$, but above $50 \%$ consistency: birth date, injury etiology, place of discharge, SL/ML and AIS. The other three (total days hospitalized, ventilatory assistance and date of neurological examination) were less than $50 \%$ consistent with the information in ISCICDS. The lowest was total days hospitalized with $39.5 \%$ consistency.

Survey responses indicate that for data regarding vertebral injury, associated injury, spinal surgery, ventilatory assistance and place of discharge, some physicians used a more detailed option instead of the ISCICDS option and were accordingly accepted as matching the information in the ISCICDS. For the data element 'date of neurological examination', the options 'days post injury' and 'days post surgery' were chosen by 12 and 10 physicians, respectively. These options are not considered to be equal to the ISCICDS option, because if the injury date or surgery date is not documented in detail as 'YYYYMMDD', it is not possible to calculate the examination date from these options. When querying the frequency of neurological examination, 20 physicians selected the ISCICDS option (at admission and discharge), whereas another 20 physicians selected a more frequent option but include at admission and discharge. Both options were accepted as consistent with the information of the ISCICDS.

\section{DISCUSSION}

To our knowledge, this is the first study to investigate the practice of collecting core data elements in individuals with SCI among physicians in China. Ideally, the minimal necessary information in relation to the first inpatient period after SCI within the ISCICDS ${ }^{2}$ should be collected during daily clinical practice. However, from the results of our survey, only 4 out of 16 data elements resembling those in the ISCICDS were collected completely consistently with the information in the ISCICDS. The other 12 data elements either were not collected at all or were not collected in accordance with the requirements of the ISCICDS 
Dates are fundamental demographic data; hence, in the ISCICDS, five data elements document time points including birth, injury, acute admission, final inpatient discharge dates and dates of neurological examinations. On the basis of these dates, age and time since injury can be calculated. In addition, time interval from injury to admission can be computed, which is important for recruitment of individuals for clinical trials. ${ }^{4}$ Unfortunately, none of these dates were consistently collected according to the requirements of the ISCICDS. The consistency percentages in descending order are injury date, final inpatient discharge date, acute admission date, birth date and date of neurological examination. Some physicians only documented year or year and month and estimated age from incomplete birth date information. Although the standardized data reporting of results from the ISCICDS ${ }^{5}$ recommend age instead of birth date to be reported for individuals with SCI, the precise age calculation requires year, month and day to be recorded. For the neurological examination date, some physicians recorded 'days post injury' or 'days post surgery' instead of 'YYYYMMDD'. If the injury date or operation date is not documented accurately, then error will be generated. Thus, this way of documenting is not recommended.

Length of stay can be calculated from the acute admission date and the final inpatient discharge date. However, total days hospitalized for acute care and rehabilitation account for the lowest consistency percentage in this survey. One possible reason may be that the recording system for acute care and rehabilitation is not always connected in China, and the length of stay is calculated for acute care and rehabilitation separately. Time periods where the patient is temporarily discharged pending re-admission would not be counted here. ${ }^{2}$ However, re-admission to acute care during the rehabilitation phase should be calculated in the length of stay and may cause confusion for some physicians. In clinical settings, it was noted that approximately $11 \%$ of SCI patients experienced re-admission to acute care during the course of rehabilitation for a variety of medical and surgical reasons. ${ }^{6}$

Data concerning gender, vertebral injury, associated injury and spinal surgery were collected consistently with the information in ISCICDS. Gender is a basic component of any medical record, whereas the other three items are also basic information in the medical history. Interestingly, some physicians documented the other three items in more detail than the information required in the ISCICDS. Two data sets are recommended for vertebral injury ${ }^{7}$ and spinal surgery ${ }^{8}$ to provide more detailed documentation of these data elements. Although ventilatory assistance is basic information for medical history, the consistency of documentation was low. The requirement of ISCICDS not only includes whether SCI individuals use ventilatory assistance but it also involves hours of utilization per day. Hence, if the data collected by the physicians did not contain the time frame, then it only partially matched the information in ISCICDS.

For injury etiology, the ICECI (International Classification of the External Cause of Injury) is the basis for registration of traumatic SCI (TSCI) in ISCICDS. Increasing familiarity with, and adherence to, the ICECI should increase the understanding of injury patterns and improve the prevention of TSCI. ${ }^{9}$ From the experience using ICECI, it can be concluded that the proportion of TSCI from land transport is decreasing/stable in developed, but increasing in developing, countries. TSCI from low falls is increasing in developed countries with aging populations, whereas high falls commonly from trees, balconies, flat roofs and construction sites are frequent occurrences in developing countries. $^{10}$ The data set for NTSCI (nontraumatic spinal cord injury $)^{11}$ is already published and is recommended to be used for documentation of NTSCI.

It is recognized that residence can affect the SCI patients' quality of life. ${ }^{12}$ According to the data from the US SCI Model System, $89.3 \%$ of all individuals with SCI are discharged to a private, non-institutional residence, whereas $6.6 \%$ are discharged to nursing homes. ${ }^{13}$ However, according to the result of this survey, some of the residency options were rarely chosen in China such as correctional institution or homeless. Therefore, it may be advised to delete the seldom chosen option(s) in future revision of the ISCICDS.

The remaining three data elements are related to the ISNCSCI (International Standards for Neurological Classification of Spinal Cord Injury). ${ }^{14}$ It is suggested that the ISNCSCI can classify the majority of SCI cases adequately. ${ }^{15}$ It should be emphasized that minimal information necessary to document neurologic status is the SL and the ML on each side of the body, as well as the AIS. ${ }^{2}$ Therefore, SL and ML, but not the NLI (neurological level of injury), as well as AIS but not the complete/incomplete injury, should be recorded. In clinical practice, the examiner could obtain NLI from the highest level of SL and ML bilaterally, as well as complete injury from AIS A and incomplete injury from AIS B to AIS D, respectively. However, we could never acquire the SL and the ML from NLI backward. In addition, despite the fact that complete injury is equal to AIS A, AIS B to AIS D could not be deduced from the incomplete injury statement merely. Furthermore, although the frequency of neurological examinations varied on the basis of the clinical status of the patient and the protocols of different institutions, ${ }^{16}$ it is suggested that it should be performed at least within $72 \mathrm{~h}$ after admission and before discharge $\mathrm{e}^{2}$ irrespective of the fact that performing it more frequently is also acceptable.

On the basis of this survey, we found that some data elements were retrieved completely consistently from the medical record, whereas other data elements were not collected in accordance with the guidelines in the ISCICDS. It is important to make sure that the data are collected correctly across centers to allow comparison and data sharing. The ultimate goal will be to include ISCICDS data elements in an electronic medical record where the appropriate instructions are available. This will ensure better understanding of the various data elements and reduce the number of mistakes made during the data collection process.

We also have to acknowledge the limitations of this study. First of all, survey respondents represent only 20 out of 31 provinces in China. Although they were from the major hospitals with SCI inpatient rehabilitation, the results of this study can only provide preliminary data. Second, the answers regarding the practice of data retrieval from the medical records were self-reported by physicians rather than confirmed by the examination of medical records. Thus, the actual data collection consistency with the information of the ISCICDS may be even worse in reality. Further studies should examine medical records from various hospitals to determine whether physicians are collecting information related to the ISCICDS as reported in this survey.

\section{CONCLUSION}

There is wide variation in the collection of data pertaining to SCI among Chinese physicians. The self-reported practice of data collection for gender, vertebral injury, associated injury and spinal surgery are completely consistent with the requirements of the ISCICDS. However, other data elements are inconsistently recorded and are therefore less comparable to the ISCICDS. It is recommended that future data collection shall be carried out using the instructions 
provided in the ISCICDS. Consistent recording of SCI core data across Chinese SCI physicians according to the ISCICDS will contribute to a uniform manner of data reporting among SCI centers in China. Core data collected in accordance with the ISCICDS will eventually lead to the development of a universal language for SCI centers in China, make worldwide comparisons possible and provide opportunities for more efficient prevention programs and SCI care.

\section{DATA ARCHIVING}

There were no data to deposit.

\section{CONFLICT OF INTEREST}

The authors declare no conflict of interest.

1 Biering-Sørensen F, Charlifue S, DeVivo M, Noonan V, Post M, Stripling $T$ et al. International Spinal Cord Injury Data Sets. Spinal Cord 2006; 44: 530-534.

2 DeVivo M, Biering-Sørensen F, Charlifue S, Noonan V, Post M, Stripling T et al. International Spinal Cord Injury Core Data Set. Spinal Cord 2006; 44: 535-540.

3 Biering-Sørensen F, Alexander MS, Burns S, Charlifue S, Devivo M, Dietz V et al. Recommendations for translation and reliability testing of international spinal cord injury data sets. Spinal Cord 2011; 49: 357-360.

4 Lee RS, Noonan VK, Batke J, Ghag A, Paquette SJ, Boyd MC et al. Feasibility of patient recruitment into clinical trials of experimental treatments for acute spinal cord injury. J Clin Neurosci 2012; 19: 1338-1343.

\section{APPENDIX}

Spinal Cord Injury Core Data Documentation Questionnaire

(The response with underline in each question is in correspondence with the information in the International Spinal Cord Injury Core Data Set)

1. In the chart, does it contain birth date information of individuals with SCI?

A. Yes, document as YYYY

B. Yes, document as YYYYMM

C. Yes, document as YYYYMMDD

D. No, never document

2. In the chart, how do you document injury date of individuals with SCI?
A. YYYY
B. YYYYMM
C. YYYYMMDD

D. Never document

3. In the chart, does it contain acute admission date information of individuals with SCI?

A. Yes, document as YYYY

B. Yes, document as YYYYMM

C. Yes, document as YYYYMMDD

D. No, never document

4. In the chart, does it contain final inpatient discharge date information of individuals with SCI?

A. Yes, document as YYYY

B. Yes, document as YYYYMM

C. Yes, document as YYYYMMDD

D. No, never document

5. From the information in the chart, how do you calculate total days hospitalized of individuals with SCI?

A. From acute admission to final discharge excluding days temporarily discharged pending readmission (that is, acute
5 DeVivo MJ, Biering-Sørensen F, New P, Chen Y. International Spinal Cord Injury Data Set. Standardization of data analysis and reporting of results from the International Spinal Cord Injury Core Data Set. Spinal Cord 2011; 49: 596-599.

6 Hammond FM, Horn SD, Smout RJ, Chen D, DeJong G, Scelza W et al. Acute rehospitalizations during inpatient rehabilitation for spinal cord injury. Arch Phys Med Rehabil 2013; 94: S98-105.

7 Dvorak MF, Wing PC, Fehlings MG, Vaccaro AR, Itshayek E, Biering-Sorensen F et al. International Spinal Cord Injury Spinal Column Injury Basic Data Set. Spinal Cord 2012; 50: 817-882.

8 Dvorak MF, Itshayek E, Fehlings MG, Vaccaro AR, Wing PC, Biering-Sorensen F et al. International Spinal Cord Injury Spinal Interventions and Surgical Procedures Basic Data Set. Spinal Cord 2015; 53: 155-165.

9 Cripps RA, Lee BB, Wing P, Weerts E, Mackay J, Brown D et al. A global map for traumatic spinal cord injury epidemiology: towards a living data repository for injury prevention. Spinal Cord 2011; 49: 493-501.

10 Lee BB, Cripps RA, Fitzharris M, Wing PC. The global map for traumatic spinal cord injury epidemiology: update 2011, global incidence rate. Spinal Cord 2014; 52: 110-116.

11 New PW, Marshall R. International Spinal Cord Injury Data Sets for non-traumatic spinal cord injury. Spinal Cord 2014; 52: 123-132.

12 Bergmark BA, Winograd CH, Koopman C. Residence and quality of life determinants for adults with tetraplegia of traumatic spinal cord injury etiology. Spinal Cord 2008; 46 : 684-689.

13 National Spinal Cord Injury Statistical Center. Spinal cord injury facts and figures at a glance. J Spinal Cord Med 2013; 36: 1-2.

14 Kirshblum SC, Burns SP, Biering-Sorensen F, Donovan W, Graves DE, Jha A et al. International standards for neurological classification of spinal cord injury (revised 2011). J Spinal Cord Med 2011; 34: 535-546.

15 Kirshblum SC, Waring W, Biering-Sorensen F, Burns SP, Johansen M, Schmidt-Read M et al. Reference for the 2011 revision of the International Standards for Neurological Classification of Spinal Cord Injury. J Spinal Cord Med 2011; 34: 547-554.

16 Consortium for Spinal Cord Medicine. Early acute management in adults with spinal cord injury: a clinical practice guideline for health-care professionals. J Spinal Cord Med 2008; 31: 403-479.

phase in orthopedic department and various rehabilitation phases altogether)

B. Calculate acute phase in orthopedic department and subsequent rehabilitation phase in the same hospital

C. Only calculate rehabilitation phase

D. Never calculate

6. In the chart, does it contain gender information of individuals with SCI?

A. Yes, document as male and female

B. No, never document

7. In the chart, how do you document injury etiology of individuals with SCI?

A. Only classify as traumatic or non-traumatic

B. Classify as traumatic or non-traumatic, traumatic injury subdivision as following order: sport/leisure, violence, transport, fall and other Traumatic according to ICECI

C. Classify as traumatic or non-traumatic, traumatic injury subdivision but not as same as the subtypes in response B

D. Never documented

8. In the chart, how do you document vertebral injury of individuals with SCI?

A. Only document as present or not

B. Document detail as response A, and also document injured vertebral level

C. Document detail as response B, and also document injured vertebral structure

D. Never document

9-1. In the chart, how do you document associated injury of individuals with SCI?

A. Only document accompanied traumatic brain injury

B. Document major associated injuries as 9-2 listed

C. Document any associated injuries

D. Never document 
9-2. In the chart, will the following items be considered as major associated injuries and documented for individuals with SCI?

\begin{tabular}{llll}
\hline Item & Yes No \\
\hline
\end{tabular}

Moderate to severe traumatic brain injury

Non-vertebral fractures requiring surgery

Severe facial injuries affecting sense organs

Major chest injury requiring chest-tube or mechanical ventilation

Traumatic amputations

Severe hemorrhage

Internal organ damage requiring surgery

10. In the chart, how do you document spinal surgery of individuals with SCI?

A. Only document as present or not

$\bar{B}$. Document detail as response $\mathrm{A}$, and also document surgical approaches

C. Document detail as response B, and also document detailed surgical methods

D. Never document

11. In the chart, how do you document ventilatory assistance of individuals with SCI?

A. Only document as present or not

B. Document utilization time per day of ventilatory assistance

C. Document utilization time per day and type of ventilatory assistance

D. Never document

12-1. In the chart, do you document place of discharge of individuals with $\mathrm{SCI}$ ?

A. Yes, document any places

B. Yes, document and describe the detailed address

C. No, never document

12-2. In the chart, will the following items be considered as place of discharge and documented for individuals with SCI?

\begin{tabular}{lll}
\hline Item & Yes & No \\
\hline
\end{tabular}

Private residence

Hospital

Nursing home
Assisted living residence Group living situation Correctional institution

Hotel or motel

Homeless

Deceased

12-3. In the chart, do you document any other place of discharge different from those in 12-2 above in individuals with SCI?

A. Document the place of discharge if present

B. Document and describe the detailed address

C. Never document

13. In the chart, do you document dates of the neurologic examinations for individuals with SCI?

A. Yes, document as YYYYMMDD

B. Yes, document as YYYYMM

C. Yes, document as YYYY

D. Days post injury

E. Days post-surgery

F. Never document

14. In the chart, how do you document injury level of individuals with SCI?

A. Only document single neurological level of injury

B. Document motor level and sensory level separately

C. Document motor level and sensory level in right and left side separately

D. Never document

15. In the chart, do you document injury severity of individuals with SCI?

A. ASIA Impairment Scale

B. Complete and incomplete injury

C. Motor complete and motor incomplete injury

D. Never document

16. In the chart, how often do you document injury level and AIS of individuals with SCI?

A. Only document at admission

B. Only document at discharge

C. Document at admission and at discharge separately

D. Document at admission, every one week after injury and at discharge

E. Never document 